\title{
Comparison between Neutrophil Lymphocyte Ratio and Derived Neutrophil Lymphocyte Ratio as the Risk Factor of COVID-19
}

\author{
Dwi Aryani ${ }^{1}$, Dea Noviana Pramatik ${ }^{2}$ \\ ${ }^{1}$ Department of Clinical Pathology, Sleman General Hospital, Yogyakarta, Indonesia. E-mail: arin8januari@gmail.com \\ ${ }^{2}$ Laboratory of Ghrasia Mental Hospital, Yogyakarta, Indonesia
}

\begin{abstract}
Coronavirus Disease 2019 (COVID-19) is an infectious disease caused by Severe Acute Respiratory Syndrome Coronavirus 2 (SARS-CoV-2). Respiratory disorders were the most common sign and symptom of COVID-19. Inflammation on SARS-CoV-2 infection is presumed to play a role in the pathogenesis of COVID-19. The Neutrophil Lymphocyte Ratio (NLR) is one of many biomarkers that has been widely used to assess the risk factors of COVID-19. The derived Neutrophil Lymphocyte Ratio (d-NLR) is a simple, inexpensive, and widely available inflammation biomarker. However, its usage for COVID-19 remains to be further studied. This study aimed to determine the NLR and d-NLR ratio as a risk factor of COVID-19. This study was a retrospective study with a study population of 84 subjects, consisting of 33 patients with positive COVID-19 and 51 patients with negative COVID-19. The result showed that the odds ratio of NLR to COVID-19 was 2.665 with the $p$-value of 0.047 and confidence interval of $95 \%$ 0.998-7.038 at cut-off $\geq 3.1$. The odd ratio of $d-N L R$ to COVID-19 was 2.808 with the $p$-value of 0.026 and confidence interval of $95 \% 1.129-7.038$ at cut-off $\geq 2.0$. In conclusion, despite a higher odd ratio of d-NLR compared to NLR, both NLR and d-NLR can be used as a biomarker for the risk factor of COVID-19.
\end{abstract}

Keywords: COVID-19, risk factor, NLR, d-NLR

\section{INTRODUCTION}

Coronavirus Disease 2019 (COVID-19) is an infectious disease caused by Severe Acute Respiratory Syndrome Coronavirus 2 (SARS-CoV-2). SARS-CoV-2 is a new type of Coronavirus that has never been identified in humans. There are at least two types of Coronavirus known to cause diseases with severe symptoms, such as Middle East Respiratory Syndrome (MERS) and Severe Acute Respiratory Syndrome (SARS). Common signs and symptoms of COVID-19 infection include acute respiratory symptoms such as fever, cough, and shortness of breath. The average incubation period is 5-6 days, with the most extended incubation period of 14 days. Severe cases of COVID-19 can cause pneumonia, acute respiratory syndrome, kidney failure, and even death. ${ }^{1}$

Coronaviruses can only reproduce through their host cells. The viruses are unable to live without a host cell. The following is the life cycle of the Coronavirus after finding host cells according to their tropism. First, the attachment of the virus to the host cell is mediated by S Protein on the surface of the virus. At the beginning of infection, the $S$ protein in SARS-CoV-2 binds to a receptor on the host cell, the Angiotensin-Converting Enzyme 2 (ACE-2). The
ACE- 2 receptors can be found on the oral and nasal mucosa, nasopharynx, lung, stomach, small intestine, large intestine, skin, thymus, bone marrow, spleen, liver, kidney, brain, pulmonary alveolar epithelial cells, small intestine enterocytes, arterial-venous endothelial cells, and smooth muscle cells. After successful entry, the translation of the gene replication from the viral RNA genome takes place. The next step is to penetrate the host cell by fusing the viral envelope with the cell membrane through a process known as Type 2 Transmembrane Serine Protease (TMPRSS2) receptor-mediated endocytosis. After the virus enters the cytoplasm, the RNA genome will come out as the envelope (coat) peels off. The viral RNA genome exits the viral membrane, partly as a template for RNA synthesis and partly as mRNA. The genome that functions as mRNA will be translated into various proteins with the help of the host cell's ribosomes. One of the proteins formed is the RNA-dependent RNA polymerase (RdRP) used for RNA replication. Another part of the RNA genome is used to synthesize negative RNA, which will be used for the template for subsequent RNA synthesis. Furthermore, maturation occurs, and positive RNA will be covered by proteins that make up the body of the virus. The glycoproteins in the newly formed viral 
envelope enter the endoplasmic reticulum membrane or Golgi cells. The nucleocapsid is then formed, composed of the RNA genome and nucleocapsid proteins. The last stage is a release, a process at which the virus is removed from the cell. ${ }^{2-5}$

Inflammation due to infectious diseases plays a vital role in pneumonia caused by viruses, including COVID-19. A severe inflammatory response contributes to a weak adaptive immune response, resulting in an immune response imbalance. Therefore, inflammatory biomarkers can indicate risk factors in COVID-19 patients. The number of leukocytes, the number of neutrophils, the number of lymphocytes, NLR, and d-NLR are the systemic inflammatory response indicators. ${ }^{3}$

Neutrophil Lymphocyte Ratio (NLR) is the ratio between the absolute value of absolute neutrophils and absolute lymphocytes. Neutrophil lymphocyte ratio has been widely used to assess risk factors for COVID-19. Several studies related to NLR have been carried out. Liu et al. in 2020 concluded that NLR was an independent risk factor for the mortality of COVID-19 patients in hospitals, especially males. The NLR assessment can help the identification of high-risk individuals with COVID-19. ${ }^{6}$

Kerboua et al. suggested that combining the two parameters as the NLR aids the formation of a solid predictive and prognostic nomogram. It is an assessment tool that enables clinicians to stratify the severity of COVID-19 on admission and guide early intervention to accelerate recovery and shorten the course of the disease to reduce shortages of medical resources and reduce mortality.

Derived Neutrophil Lymphocyte Ratio (d-NLR) is absolute neutrophils count divided by leukocytes minus absolute neutrophils. There have not been many studies on d-NLR as a risk factor for COVID-19. However, similar to NLR biomarkers, $d-N L R$ is also a simple, inexpensive, and widely available inflammatory biomarker that is widely investigated as a helpful predictor for the prognosis of patients with viral pneumonia.

The neutrophil-lymphocyte ratio and derived neutrophil-lymphocyte ratio were not statistically different between patients with benign, premalignant and malignant laryngeal neoplasms. Both neutrophil-lymphocyte ratio and derived neutrophil-lymphocyte ratio were predictive factors for stage, lymph node metastasis, and distant metastasis. Patients with high neutrophil-lymphocyte ratio value $(\geq 4)$ had a poor prognosis when compared with patients with low neutrophil-lymphocyte ratio value (5 year, overall survival: $69.0 \%$ vs. $31.1 \%$, $p<0.001 ; 5$ year, disease free survival: $70.0 \%$ vs. $32.7 \%$, $\mathrm{p}<0.001 ; 5$ year, locoregional recurrence free survival:
$69.7 \%$ vs. $32.0 \%$, p < 0.001). Furthermore, NLR was an independent prognostic factor for 5 year: overall survival (hazard ratio $=2.396 ; 95 \%$ CI 1.408-4.077; $p=0.001$ ), disease free survival (hazard ratio $=2.246$; 95\% CI 1.322-3.816; $p=0.006)$ and locoregional recurrence free survival (hazard ratio $=2.210 ; 95 \% \mathrm{CI}$ 1.301-3.753; $p=0.003){ }^{8}$. Li et al. found that dNLR prior to treatment and BI-RADS classification are independent prognostic factors in patients with HER2 + breast cancer receiving neoadjuvant chemotherapy. ${ }^{9}$

This study aimed to determine the comparison between NLR and $d-N L R$ as a risk factor for COVID-19.

\section{METHODS}

This research was performed at the Clinical Pathology Installation of Sleman Hospital in June 2020. This research was a retrospective study using data from the patient medical record. Inclusion criteria were patients suspected with COVID-19 from whose a swab specimen is taken for PCR test. Exclusion criteria were patients suspected of COVID-19 and with incomplete laboratory data. This research was approved by the Health Research Ethics Committee of the Sleman Hospital with number $180 / 2282$. In addition, a hematological test was carried out with Sysmex XN-L.

The primary characteristics of the research subjects were presented descriptively in means and standard deviations if the data distribution was normal. However, data were delivered in median, maximum, and minimum if the distribution was not normal. The normality test for continuous data was carried out using the Kolmogorov-Smirnov test, with a significance value of $p<0.05$. Unpaired T-test was used for the different tests if the numerical variables and data distribution were standard. Still, Mann-Whitney U-test was used if the data distribution was not normal or categorical variables. The $p$-value $<0.05$ indicated a significant difference between variables. Bivariate analysis was carried out using Chi-Square. The data were then processed using SPSS 23.

\section{RESULTS AND DISCUSSIONS}

In June 2020, 114 subjects who underwent swab sampling for PCR test of SARS-CoV-2 were found. A total of 30 subjects were excluded from the analysis due to incomplete medical records. In this study, 84 subjects met the inclusion and exclusion criteria. Characteristics of research subjects such as gender, age, and laboratory parameters can be seen in Table 1. 
Table 1. Characteristics of research subjects

\begin{tabular}{lc}
\hline \multicolumn{1}{c}{ Variable } & Value \\
\hline Gender $\mathbf{n}(\%)$ & \\
Male & $46(54.8 \%)$ \\
Female & $38(45.2 \%)$ \\
Age years, mean $(\mathrm{SD})$ & $42.4(16.356)$ \\
Hemoglobin $(\mathrm{Hb}) \mathrm{gr} / \mathrm{dL}$, median (min-max) & $13.90(10.2-17.3)$ \\
Leukocyte count $10^{3} / \mu \mathrm{L}$, mean (SD) & $8.25(2.55)$ \\
Absolute neutrophil $10^{3} / \mu \mathrm{L}$, mean (SD) & $5.08(1.90)$ \\
Absolute lymphocyte $10^{3} / \mu \mathrm{L}$, median (min-max) & $2.15(0.41-8.51)$ \\
Absolute monocyte $10^{3} / \mu \mathrm{L}$, median (min-max) & $0.48(0.12-1.88)$ \\
Absolute eosinophil $10^{3} / \mu \mathrm{L}$, median (min-max) & $0.09(0-1.1)$ \\
Absolute basophil $10^{3} / \mu \mathrm{L}$, median (min-max) & $0.03(0-0.11)$ \\
Erythrocyte count $10^{3} / \mu \mathrm{L}$, mean (SB) & $4.97(0.56)$ \\
Platelet count $10^{3} / \mu \mathrm{L}, \mathrm{median}$ (min-max) & $295(77-690)$ \\
NLR, median (min-max) & $2.16(0.38-6.21)$ \\
d-NLR, median $(\mathrm{min}-\mathrm{max})$ & $1.66(0.33-6.21)$ \\
\hline
\end{tabular}

Table 2. Difference test of research variables between subjects with positive and negative PCR test results

\begin{tabular}{lccc}
\hline \multicolumn{1}{c}{ Variable } & Positive PCR $(\mathbf{n}=\mathbf{3 3})$ & Negative PCR $(\mathbf{n}=\mathbf{5 1})$ & $\mathbf{p}$ \\
\hline Age (years) & $38.27(16.333)$ & $45.08(15.96)$ & $0.064^{*}$ \\
Hemoglobin $(\mathrm{Hb})(\mathrm{gr} / \mathrm{dL})$ & $13.60(10.60-17.30)$ & $14.10(10.20-16.20)$ & $0.555^{* *}$ \\
Leukocyte count $10^{3} / \mu \mathrm{L}$ & $8.63(2.35)$ & $8.01(2.66)$ & $0.282^{*}$ \\
Absolute neutrophil $10^{3} / \mu \mathrm{L}$ & $5.59(1.76)$ & $4.74(1.93)$ & $0.045^{*}$ \\
Absolute lymphocyte $10^{3} / \mu \mathrm{L}$ & $1.83(1.20-6.15)$ & $2.18(0.41-8.51)$ & $0.108^{* *}$ \\
Absolute monocyte $10^{3} / \mu \mathrm{L}$ & $0.57(0.33-1.88)$ & $0.44(0.12-167)$ & $0.001^{* *}$ \\
Absolute eosinophil $10^{3} / \mu \mathrm{L}$ & $0.06(0-1.1)$ & $0.03(0-0.11)$ & $0.037^{*}$ \\
Absolute basophil $10^{3} / \mu \mathrm{L}$ & $0.03(0.01-0.1)$ & $0.03(0-0.11)$ & $0.558^{* *}$ \\
Erythrocyte count $10^{3} / \mu \mathrm{L}$ & $4.99(0.63)$ & $4.95(0.52)$ & $0.765^{*}$ \\
Platelet count $10^{3} / \mu \mathrm{L}$ & $296(129-676)$ & $294(77-690)$ & $0.819^{* *}$ \\
NLR (T) & $0.41(0.18)$ & $0.28(0.25)$ & $0.012^{*}$ \\
d-NLR (T) & $0.28(0.19)$ & $0.16(0.25)$ & $0.031^{*}$ \\
\hline
\end{tabular}

Note: $(\mathrm{T})=$ data transformation with $\log 10,\left({ }^{*}\right)=$ T-test,$\left({ }^{*}\right)=$ Mann-Whitney

The results showed that the research subjects were 46 (54.8\%) male and 38 (45.2\%) female subjects. The age of the youngest issues was three years, and the oldest was 83 years, with a mean of 42.5 years. This result was following the research of Usul et al., which involved 177 (62.8\%) male and 105 (37.2\%) female subjects and obtained the mean age of $47.6 \pm 16.9$ years. ${ }^{10}$ Liao et al. reported that $206(54 \%)$ and 174 (46\%) of the study subjects were male and female, respectively. ${ }^{11}$

Liao et al. in his study included 380 study subjects and reported laboratory test results as follows: median of leukocyte counts of 5.89 (4.40-8.27) $\times 10^{3} / \mu \mathrm{L}$; median of absolute neutrophils of $4.19(2.71-6.37) \times 10^{3} / \mu \mathrm{L} ;$ median of absolute lymphocytes of $0.95(0.59-1.47) \times 10^{3} / \mu \mathrm{L}$; median of absolute monocyte of $0.47(0.30-0.62) \times 10^{3} / \mu \mathrm{L}$; median of eosinophil absolute of $0.03(0.01-0.09) \times 10^{3} / \mu \mathrm{L}$; median of absolute basophils of $0.01(0.01-0.03) \times 10^{3} / \mu \mathrm{L}$; median $\mathrm{Hb}$ of 11.9 (10.7-13.1) gr/dL; median platelet

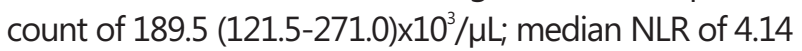
(2.25-9.08). ${ }^{11}$ In addition, there were close similarities between characteristics of routine bood test results in this study and research by Liao et al.

This study carried out a different test of research variables between 33 subjects with positive and 51 subjects with negative PCR test results.

Table 2 showed the different test results between subjects with positive and negative PCR test that has a difference in absolute neutrophils, absolute monocytes, absolute eosinophils, NLR, and d-NLR Contrastingly, there was no significant difference in age, hemoglobin, total leukocytes, absolute lymphocytes, absolute basophils, erythrocyte 
counts, and platelet counts between subjects with positive PCR and negative PCR tests.

Usul et al. reported in his study that there was a significant difference in hemoglobin, leukocyte count, platelet count, absolute neutrophils and NLR, but no significant difference of age and total lymphocytes was found between 163 subjects with positive COVID-19 and 119 subjects with negative COVID-19. ${ }^{10}$ A study by Nalbat et al. involving 54 research subjects with positive COVID-19 and 26 subjects with negative COVID-19 reported a significant difference of hemoglobin, absolute lymphocytes, and NLR but no significant difference of age and platelet count was found between subjects with positive and negative COVID-19. ${ }^{12}$

A study by Yan et al. on 964 survivors and 40 non-survivors of patients confirmed with COVID-19 showed a significant difference in age, leukocyte count, platelet count, absolute neutrophils, and absolute lymphocytes NLR. However, there was no significant difference in hemoglobin. ${ }^{13}$ A study by Yang et al. on positive COVID-19 patients, 69 subjects experienced mild symptoms, and 24 subjects experienced severe symptoms. In addition, a significant difference in age, leukocyte count, absolute neutrophils, absolute monocytes, absolute lymphocytes, NLR, and d-NLR was found. ${ }^{5}$
There were variations of significance in the variables between this study and previous studies. This might be influenced by the number of research subjects and the degree of disease severity. However, there was a significant difference of NLR variable in this study and previous studies, suggesting a consistent considerable difference in both positive and negative COVID-19 cases or survivor and non-survivor COVID-19 patients.

A bivariate analysis was carried out to determine the risk factors for the incidence of COVID-19. In this bivariate analysis, cut-off $\geq 3.1$ and cut-off $\geq 2.0$ were used for NLR and $d-N L R$, respectively. The Odds Ratio (OR) of each variable was then calculated (Table 3).

According to the results of bivariate analysis, the variables that showed a significant $O R$ were absolute monocytes, absolute lymphocytes, NLR, and d-NLR. Absolute monocytes showed cut-off $\geq 0.5 \times 10^{3} / \mu \mathrm{L}$ and OR 3.667; absolute lymphocytes showed a cut-off $<2.1 \times 10^{3} / \mu \mathrm{L}$ and OR of 1.386; NLR showed a cut-off $\geq 3.1$ and OR of 2.665 and $d-N L R$ showed a cut-off $\geq 2.0$ and OR of 2.808 .

A study by Yang et al. about the risk of death in COVID-19 patients showed that only NLR had a significant hazard ratio against death in COVID-19 patients by 2.462 times. Contrastingly, no significant

Table 3. Bivariate Analysis of COVID-19

\begin{tabular}{|c|c|c|c|c|c|c|}
\hline \multirow{2}{*}{ Variable } & & \multicolumn{2}{|c|}{ PCR } & \multirow{2}{*}{ OR } & \multirow{2}{*}{ CI 95\% } & \multirow{2}{*}{$\mathbf{p}$} \\
\hline & & Pos & $\mathrm{Neg}$ & & & \\
\hline \multirow[t]{2}{*}{ Gender } & Male & 19 & 27 & 1.206 & $0.499-2.916$ & 0.677 \\
\hline & Female & 14 & 24 & & & \\
\hline \multirow[t]{2}{*}{ Hemoglobin (gr/dL) } & $<12$ & 4 & 6 & 1.034 & $0.269-3.984$ & 0.961 \\
\hline & $\geq 12$ & 29 & 45 & & & \\
\hline \multirow[t]{2}{*}{ Leukocyte count $\left(10^{3} / \mu \mathrm{L}\right)$} & $<5$ & 0 & 4 & 1.702 & $1.147-2.045$ & 0.099 \\
\hline & $\geq 5$ & 33 & 47 & & & \\
\hline \multirow[t]{2}{*}{ Absolute neutrophil $\left(10^{3} / \mu \mathrm{L}\right)$} & $\geq 4.5$ & 23 & 25 & 2.392 & $0.950-6.022$ & 0.061 \\
\hline & $<4.5$ & 10 & 26 & & & \\
\hline \multirow[t]{2}{*}{ Absolute monocyte $\left(10^{3} / \mu \mathrm{L}\right)$} & $\geq 0.5$ & 22 & 18 & 3.667 & $1.455-9.237$ & $0.005^{*}$ \\
\hline & $<0.5$ & 11 & 33 & & & \\
\hline \multirow[t]{2}{*}{ Absolute lymphocyte $\left(10^{3} / \mu \mathrm{L}\right)$} & $<2.1$ & 13 & 29 & 1.386 & $0.157-0.949$ & $0.036^{*}$ \\
\hline & $\geq 2.1$ & 20 & 22 & & & \\
\hline \multirow[t]{2}{*}{ Platelet count $\left(10^{3} / \mu \mathrm{L}\right)$} & $<150$ & 1 & 2 & 0.766 & $0.067-8.787$ & 0.830 \\
\hline & $\geq 150$ & 32 & 49 & & & \\
\hline \multirow[t]{2}{*}{ Erythrocyte count $\left(10^{3} / \mu \mathrm{L}\right)$} & $<4.5$ & 7 & 6 & 2.019 & $0.613-6.654$ & 0.242 \\
\hline & $\geq 4.5$ & 26 & 45 & & & \\
\hline \multirow[t]{2}{*}{ NLR } & $\geq 3.1$ & 13 & 10 & 2.665 & $0.998-7.119$ & $0.047^{\star}$ \\
\hline & $<3.1$ & 20 & 41 & & & \\
\hline \multirow[t]{2}{*}{ d-NLR } & $\geq 2.0$ & 17 & 14 & 2.808 & $1.120-7.038$ & $0.026^{*}$ \\
\hline & $<2.0$ & 16 & 37 & & & \\
\hline
\end{tabular}


hazard ratio values were found in leukocyte count and d-NLR. The cut-off of NLR and d-NLR in a study by Yang was $\geq 3.3$ and $\geq 2.8$, respectively. ${ }^{5} A$ study by Liu et al. involved 245 COVID-19 patients and evaluated OR for death, showed that only absolute neutrophils had a significant $O R$ for death. In contrast, total lymphocytes, hemoglobin, and platelets had no significant OR for death. ${ }^{6}$ The results of this study are that NLR and d-NLR in patients with COVID-19 are different from Yang et al. The cut-off NLR and d-NLR used are different, and the research subjects are suspected of COVID-19, in a Yang et al. study that was COVID-19.

Research at a hospital in China's Hubei province on COVID-19 patients consisting of those with 32 moderate and 31 severe cases showed that NLR with a cut-off $>4.7$ was a risk factor for disease severity. ${ }^{14}$ A study by Cox et al. showed a cut-off $>2.0$ and hazard ratio 1.74 (95\% CI 1.29-2.35], p < 0.001) in multivariate analysis and hazard ratio 1.64 [1.17-2.29], $p=0.004)$ on mortality in patients with esophageal cancer. ${ }^{15}$

\section{CONCLUSIONS AND SUGESSTIONS}

There was a significant difference in the d-NLR and NLR values in patients with positive and negative COVID-19. Increased d-NLR and NLR values were risk factors for COVID-19 in patients with suspected COVID-19. An increase in d-NLR led to a higher odds ratio compared to the rise in NLR.

The derived neutrophil-lymphocyte ratio in suspect COVID-19 patients became one of many parameters considered a risk factor of COVID-19.

\section{REFERENCES}

1. Kementerian Kesehatan Republik Indonesia. Pedoman pencegahan dan pengendalian Coronavirus Disease (COVID-19). Jakarta, Kemenkes, 2020; 17.

2. Perhimpunan Dokter Paru Indonesian. Pneumonia COVID-19 diagnosis dan penatalaksanaan di Indonesia. Jakarta, Perhimpunan Dokter Paru Indonesia, 2020; 4-7.

3. Wiersinga JW, Rhodes AJ, Cheng MD, Peacock SJ, Prescott HC, Pathophysiology, transmission, diagnosis, and treatment of Coronavirus Disease 2019 (COVID-19) a review. JAMA, 2020; 324(8): 782-793.
4. Ilmu Penyakit Dalam RS Dr M. Hoesin/FK UNSRI Palembang. Praktis COVID-19, Palembang, 2020; 7-8.

5. Yang AP, Liu JP, Tao WQ, Li Hiu-ming. The diagnostic and predictive role of NLR, d-NLR, and PLR in COVID-19 patients. International Immunopharmacology, 2020; 84: $2-4$

6. Liu $Y$, Du $X$, Chen $X$, Jin $Y$, Peng $L$, et al. Neutrophil-to-lymphocyte ratio as an independent risk factor for mortality in hospitalized patients with COVID-19. Journal of Infection, 2020; 81: 6-12.

7. Kerboua KE. NLR: A cost-effective nomogram to guide therapeutic interventions in COVID19. Immunological Investigation, 2020; 50(8): 1-9.

8. Eskiizmir G, Uz U, Onur G, Ozgur B, Cicriki GK, et al. The evaluation of pre-treatment neutrophil-lymphocyte ratio and derived neutrophil-lymphocyte ratio in patients with laryngeal neoplasms. Brazilian Journal of Otorhinolaryngology, 2019; 85(5): 578-587.

9. Li $Y$, Shao $Y$, Bai $L$, Zhou $X$. Increased derived neutrophil-to-lymphocyte ratio and breast imaging-reporting and data system classification predict poor survival in patients with non-distant metastatic HER2+ breast cancer treated with neoadjuvant chemotherapy. Shanxi, 2018; 10: 3841-3847.

10. Usul E, San I, Bekgos B, Shahin A. The role of hematological parameters in COVID-19 patients in the emergency room. Biomark Med, 2020; 317: 2-7.

11. Liao $D$, Zhou $F$, Luo $L$, Xu M, Wang $H$, et al. Haematological characteristics and risk factors in the classification and prognosis evaluation of COVID-19: A retrospective cohort study. Lancet Haematology, 2020; 7: 671-78.

12. Nalbant A, Kay T, Varin C, Cinemre S. Can the Neutrophil/Lymphocyte Ratio (NLR) have a role in the diagnosis of Coronavirus 2019 Disease (COVID-19)?. Revista da Associação Médica Brasileira, 2020; 66(6): 746-751.

13. Yan $X$, Li F, Wang $X$, Yan J, Zhui F, et al. Neutrophil to lymphocyte ratio as a prognostic and predictive factor in patients with Coronavirus Disease 2019: A retrospective cross-sectional study. Journal of Medical Virology, 2020; 1-9.

14. Pimantel GD, Dela Vega, Laviano A. High neutrophil to lymphocyte ratio as a prognostic marker in COVID-19 patients. European Society for Clinical Nutrition and Metabolism. Roma, Elsevier Ltd, 2020; 1-2.

15. Cox S, Hurt C, Grenadier T, Mukherjee S, Bridgewater J, et al. The prognostic value of derived neutrophil to lymphocyte ratio in oesophageal cancer treated with definitive chemoradiotherapy. London, Elsevier Ireland Ltd, 2017; 154-159. 